\title{
SALUTO DEL PRESIDENTE DELL'ASSOCIAZIONE ITALIANA DI MECCANICA TEORICA E APPLICATA (AIMETA)
}

\author{
PAOLO LUCHINI (*)
}

Con grande piacere ringrazio l'Istituto Lombardo Accademia di Scienze e Lettere che ospita questo convegno congiunto con l'AIMETA, Associazione Italiana di Meccanica Teorica e Applicata. Molti dei presenti ben conoscono l'AIMETA. Come si legge nel primo numero della rivista Meccanica (1966), l'AIMETA riunisce i cultori della Meccanica nei suoi vari indirizzi: teorico, sperimentale, tecnico, e applicativo. Ognuno ha naturalmente mentalità consona all'indirizzo nel quale si svolgono le sue ricerche e la sua attività didattica e professionale, ma per tutti l'interesse verte sulla Meccanica, scienza ampissima, multiforme, in continuo sviluppo, "paradiso delle scienze matematiche" come diceva Leonardo, ma anche fondamento di ogni scienza fisica. Meccanica intesa in senso lato che è, come diceva Newton, "scientia motum, qui ex viribus quibuscumque resultant, et virium quae ad motus quoscunque requiruntur." Più generalmente, come diceva Kirchhoff, è la "descrizione completa dei movimenti che avvengono in natura" o, col linguaggio di Einstein, "geometria dello spaziotempo". In questo senso, nell'AIMETA è difficile segnare il confine fra Meccanica razionale e Fisica matematica, fra Meccanica applicata, Scienza delle costruzioni, Idraulica, Aerodinamica. Ma l'AIMETA si apre anche verso i ricercatori che operano in campi attigui a quelli che le sono propri in senso stretto. I Gruppi di Biomeccanica e di Meccanica dei materiali sono un segno dell'apertura culturale dell'asso-

(*) Presidente AIMETA, Università di Salerno - DIIN, Fisciano (SA), Italia. E-mail: luchini@unisa.it 
ciazione. Attraverso convegni generali biennali, attraverso la rivista Meccanica, e attraverso gruppi di interesse che organizzano convegni specifici, l'associazione si propone di stabilire contatti fra ricercatori che operano nel medesimo indirizzo, e soprattutto in indirizzi diversi, su temi, anche ristretti, nei quali possano confluire conoscenze ed esperienze diverse. In ambito internazionale l'AIMETA si colloca nella IUTAM, International Union of Theoretical and Applied Mechanics, organismo che raccoglie associazioni affiliate di 50 nazioni. La IUTAM, fondata nel 1922 intorno a questioni principalmente di Idrodinamica ed Aerodinamica, si pone oggi come collegamento mondiale tra le associazioni nazionali di Meccanica dei Fluidi e dei Solidi, principalmente attraverso la sua conferenza generale quadriennale. L'organizzazione della conferenza generale della IUTAM nel 2020 è stata affidata all'Italia, grazie anche all'interessamento dell'AIMETA, e in particolare a Milano, sede dell'Istituto Lombardo. E non a caso, come si può intuire dalla data di fondazione sopra riportata, il convegno di oggi cade nell'anno in cui l'AIMETA celebra il suo cinquantenario. I rapporti tra AIMETA e Istituto Lombardo Accademia di Scienze e Lettere sono sempre stati molto stretti: tra l'altro l'Istituto Lombardo cortesemente ospita gli archivi dell'AIMETA, e cinque membri dell'Istituto Lombardo sono stati presidenti AIMETA:

- Bruno Finzi (1966-69). Primo presidente

- Giovanni Bianchi (1982-85)

- Giulio Maier (1986-89)

- Carlo Cercignani (1990-93)

- Carlo Cinquini (2010-2013)

È quindi con grande onore che, nell'ambito delle celebrazioni del cinquantenario alle quali sarà anche dedicato un numero speciale della rivista Meccanica, saluto e ringrazio tutti i partecipanti a questo convegno congiunto che sta per iniziare. 